\title{
Impacto do trabalho e satisfação da equipe multiprofissional de um hospital psiquiátrico
}

\author{
Satisfaction in, and impact of work on, the multidisciplinary team in a psychiatric hospital \\ Impacto del trabajo y satisfacción del equipo multidisciplinario en un hospital psiquiátrico
}

\author{
Giselle Cristina Dias'; Antonia Regina Ferreira Furegato"
}

\begin{abstract}
RESUMO
Objetivo: identificar a satisfação e o impacto do trabalho entre profissionais da equipe multiprofissional de um hospital psiquiátrico. Método: estudo exploratório descritivo, quantitativo, do tipo transversal, realizado em 2013, numa cidade do interior de São Paulo, junto à equipe multiprofissional de um hospital psiquiátrico. A população foi constituída pelos 138 membros da equipe multiprofissional, sendo a amostra de 116 participantes. Foram utilizados para a coleta dos dados: Roteiro para entrevista com os sujeitos, Escala de Avaliação da Satisfação da Equipe em Serviços de Saúde Mental abreviada e Escala de Avaliação do Impacto do Trabalho em Serviços de Saúde Mental. Resultados: o escore médio de satisfação foi 3,4, situado na indiferença, indicando níveis baixos de satisfação; entretanto, o índice de sobrecarga foi 1,9, apontando grau médio de sobrecarga. Conclusão: baixos níveis de satisfação mostram a necessidade de revisão dos projetos institucionais.

Palavras-chave: Enfermagem psiquiátrica; satisfação no emprego; saúde mental; saúde do trabalhador.
\end{abstract}

\section{ABSTRACT}

Objective: to identify job satisfaction and impact of work among members of a multidisciplinary team at a psychiatric hospital. Method: an exploratory, quantitative, cross-sectional descriptive study was conducted in 2013, with the multidisciplinary team of a psychiatric hospital in São Paulo city. The population comprised 138 members of the multidisciplinary team, of whom 116 formed the sample. Data were collected by: interview script, a Mental Health Service Team Satisfaction Assessment Scale and a Mental Health Service Work Impact Assessment Scale. Results: average satisfaction score was 3.4, indicating indifference, i.e., low levels of satisfaction. Also, the overwork index was 1.9, indicating a medium degree of overwork. Conclusion: it was concluded that low levels of satisfaction show the need to review institutional projects.

Keywords: Psychiatric nursing; job satisfaction; mental health; occupacional health

\section{RESUMEN}

Objetivo: identificar la satisfacción y el impacto en el trabajo y entre profesionales del equipo multidisciplinario de un hospital psiquiátrico. Método: estudio exploratorio descriptivo, de abordaje cuantitativo, de tipo transversal, realizado en 2013, en una ciudad del interior del Estado de São Paulo, junto al equipo multidisciplinario de un hospital psiquiátrico. La población se compone de 138 miembros del equipo multidisciplinario; la muestra fue de 116 personas. Se utilizaron, para la recolección de los datos: guión para la entrevista, Escala de Evaluación de la Satisfacción del Equipo en Servicios de Salud Mental de forma abreviada y Escala de Evaluación del Impacto del Trabajo en Servicios de Salud Mental. Resultados: la puntuación media de satisfacción fue de 3,4, lo que significa indiferencia e indica bajos niveles de satisfacción; sin embargo, el índice de sobrecarga fue de 1,9, lo que indica grado medio de sobrecarga. Conclusión: se concluye que los bajos niveles de satisfacción muestran la necesidad de revisión de los proyectos institucionales. Palabras clave: Enfermería psiquiátrica; satisfacción en el trabajo; salud mental; salud laboral.

\section{INTRODUÇÃO}

A saúde é o resultado do gerenciamento adequado das áreas físicas, emocionais, sociais, profissionais, intelectuais e espirituais e a qualidade de vida está relacionada a manter-se o equilíbrio interior diante das situações do dia a dia ${ }^{1-5}$. Evidentemente, as pessoas trabalham visando retorno financeiro, porém o desempenho de cada indivíduo será maior se ele estiver motivado e comprometido com o trabalho.

A realização profissional pode estar relacionada diretamente com a satisfação no trabalho. Isso tem im- plicações com a supervisão, com benefícios, pagamento, oportunidades, políticas da organização e do controle que os trabalhadores possuem sobre suas condições de vida e de trabalho. Também sinaliza que estar satisfeito é um fator importante de realização profissional e pode ser entendido como fator de proteção à saúde mental do trabalhador ${ }^{6-8}$.

O ambiente hospitalar, por si só, pode ser considerado um fator estressor, por possuir condições de insalubridade e periculosidade se comparado a outros tipos de serviços ${ }^{9}$. 
Diante do exposto, o presente estudo teve como objetivo identificar a satisfação e o impacto do trabalho em profissionais da equipe multiprofissional de um hospital psiquiátrico.

\section{REVISÃo DE LITERATURA}

Sabe-se que as profissões da área de saúde estão em terceiro lugar como causadoras de estresse. A enfermagem está na quarta posição como a mais estressante ${ }^{10}$.

Como o trabalho da equipe de enfermagem em um hospital é escalonado em turnos, os trabalhadores sofrem prejuízos, com relação à participação em eventos sociais, repercutindo na saúde mental dos trabalhadores de saúde $\mathrm{e}^{10,11}$.

Os profissionais da saúde convivem com ansiedade, dor, sofrimento e sentimentos negativos dos pacientes. A angústia experimentada pela equipe é grande, o que gera tensão psíquica. Em função disso, é necessário dar devida atenção ao aspecto psicológico da equipe, para possibilitar o enfrentamento a fatores e vivências desgastantes no local de trabalho ${ }^{6-12}$.

\section{MetOdOLOGiA}

Trata-se de um estudo descritivo, do tipo transversal, realizado numa instituição de assistência psiquiátrica, de uma cidade do interior do Estado de São Paulo, em 2013. Dos 138 profissionais pertencentes à equipe multiprofissional da instituição estudada, 116 profissionais participaram da pesquisa. No entanto, quatro profissionais não foram entrevistados por estarem de férias, dois se recusaram a participar, cinco estavam afastados e 11 sujeitos foram excluídos por estarem há menos de seis meses na instituição, totalizando 22 sujeitos excluídos do estudo.

Participaram deste estudo 116 profissionais da instituição, sendo 11 médicos, oito enfermeiros, quatro psicólogos, um fisioterapeuta, três terapeutas ocupacionais, três assistentes sociais, um dentista, um nutricionista, dois educadores físicas, um farmacêutico, 69 técnicos de enfermagem e 12 auxiliares de enfermagem. O critério de seleção foi atuar há pelo menos seis meses na instituição como membro da equipe de profissionais de nível superior ou como auxiliar e técnico de enfermagem e não estar afastado por férias ou licenças.

Foram utilizados como instrumentos de investigação: o roteiro para entrevista com profissionais de saúde mental (REPSM), a Escala de Avaliação da Satisfação da Equipe em Serviços de Saúde Mental (SATIS-BR abreviada) e a Escala de Avaliação do Impacto do Trabalho em Serviços de Saúde Mental (IMPACTO-BR). O roteiro para entrevista foi construído pelas pesquisadoras, com base na literatura sobre o tema da pesquisa. É composto por 14 itens, com questões de identificação dos sujeitos, nível de escolaridade, atuação em serviço de saúde mental e outros dados sociodemográficos. As escalas de avaliação integram um conjunto de escalas para avaliação de serviços de saúde mental, elaborados pela Divisão de Saúde Mental da Organização Mundial de Saúde (OMS) e foram validadas para o Brasil ${ }^{12}$.

A Escala SATIS-BR contém 32 questões quantitativas que avaliam o grau de satisfação da equipe em relação aos serviços oferecidos e às condições de trabalho na instituição em que atuam. Há três questões descritivas que complementam essas informações e referem-se à opinião da equipe sobre os serviços. As respostas estão dispostas em escala ordinal (tipo Likert) de cinco pontos, que vai de Muito insatisfeito (1) a Muito satisfeito (5). Foram identificados quatro fatores: Fator 1 - Grau de satisfação da equipe em relação à qualidade dos serviços oferecidos; Fator 2 - Grau de satisfação da equipe em relação a sua participação no serviço; Fator 3 - Grau de satisfação da equipe em relação às condições de trabalho; Fator 4 - Grau de satisfação da equipe em relação ao seu relacionamento com os colegas de trabalho e com seus superiores no serviço ${ }^{12}$.

A Escala IMPACTO-BR contém 18 questões quantitativas que avaliam o grau de sobrecarga sentido pelos profissionais de saúde mental que trabalham diariamente com portadores de sofrimento psíquico e três questões descritivas complementares. As respostas estão dispostas em uma escala ordinal (tipo Likert) de cinco pontos. A escala apresentou, com base na análise de suas qualidades métricas, a existência de três fatores: Fator 1 - Sobrecarga referente aos efeitos sentidos pela equipe em relação a sua saúde física e mental; Fator 2 - Sobrecarga referente ao impacto do trabalho sobre o funcionamento da equipe; Fator 3 - Sobrecarga referente às repercussões emocionais do trabalho ${ }^{12}$.

O projeto foi aprovado pelo Comitê de Ética em Pesquisa da Escola de Enfermagem de Ribeirão Preto da Universidade de São Paulo (212/2012). Obteve-se consentimento e autorização da coordenação administrativa da instituição. Os participantes foram informados sobre a natureza, os objetivos e os procedimentos durante a pesquisa. Todos os que concordaram em participar da pesquisa assinaram o Termo de Consentimento Livre e Esclarecido.

Utilizou-se o programa Statistic Package for Social Sciences (SPSS), versão 10.0, para as análises estatísticas. Realizou-se a análise descritiva e a análise inferencial. Utilizou-se o teste Fisher para calcular as associações ${ }^{13}$. Calculou-se o escore médio geral de satisfação e de sobrecarga e os valores referentes às subescalas. Para comparação entre os resultados obtidos entre os fatores de satisfação e sobrecarga, utilizou-se a Correlação de Spearman; realizou-se, também, a correção de Bonferroni para as comparações múltiplas. A comparação dos escores de satisfação e sobrecarga no trabalho foi realizada pelo Teste de Kruskal-Wallis ${ }^{13}$. Para todos os testes estatísticos foram definidos, como nível de significância, os valores de $p<0,05$. 


\section{RESULTADOS}

\section{Caracterização da amostra}

Dos 116 sujeitos do estudo, 74(63,8\%) são do sexo feminino, 40(54\%) são solteiras e há predominância de sujeitos do sexo masculino que são casados 25(59,5\%). A maioria da amostra - 60(51,7\%) - não tem filhos, o sexo masculino representou $21(50 \%)$ dos que possuem filhos. A maior proporção dos sujeitos possui o nível médio $62(53,4 \%)$ e $54(46,6 \%)$ possuem nível superior. A idade dos sujeitos variou de 20 a 74 anos. Cerca de $1 / 3$ dos participantes trabalhava há até 2 anos na instituição, outro terço, de 3 a 10 anos e outro terço acima de 11 anos, variando de 1 a 38 anos trabalhados.

\section{Satisfação no trabalho}

Os dados obtidos, através da Escala de Avaliação SATIS-BR, permitiram calcular os escores de satisfação por sujeito e o escore médio de satisfação para o conjunto da amostra. O escore geral de satisfação variou de 2,2 a 4,8 e a média dos escores foi 3,4, valor situado em Indiferente, indicando índices baixos de satisfação.

O fator de maior satisfação foi Relacionamentos, apresentando escore médio de 3,7, seguido pelos fatores Qualidade do serviço e Condições de trabalho com escores médios de 3,4. O fator de menor satisfação foi Participação no serviço, conforme demonstrado na Tabela 1.

TABELA 1: Média e desvio-padrão dos escores de satisfação geral e por fatores dos profissionais de um hospital psiquiátrico. Franca, São Paulo, 2013. ( $\mathrm{N}=116)$

\begin{tabular}{lccc}
\hline \multicolumn{1}{c}{ SATIS-BR } & $\begin{array}{c}\text { Escore } \\
\text { médio }\end{array}$ & $\begin{array}{c}\text { Desvio } \\
\text { padrão }\end{array}$ & Variação \\
\hline Satisfação geral & 3,4 & 0,6 & $2,2-4,8$ \\
Qualidade do serviço (F1) & 3,4 & 0,7 & $2,2-4,8$ \\
Participação no serviço (F2) & 3,2 & 0,7 & $1,3-5,0$ \\
Condições de Trabalho (F3) & 3,4 & 0,6 & $2,2-4,9$ \\
Relacionamentos (F4) & 3,7 & 0,8 & $2,0-5,0$ \\
\hline
\end{tabular}

Considerando-se as três questões com maiores escores de satisfação, observou-se bom relacionamento da equipe $(4,1)$, confiabilidade no serviço e autoconfiança da equipe, expressos na valorização do relacionamento com os colegas de trabalho, na recomendação do serviço a amigos e familiares $(3,9)$ e no grau de responsabilidade da equipe no serviço $(3,8)$.

Os benefícios do trabalho $(2,3)$ foram destaque negativo entre os escores de satisfação. Entre outros aspectos de menor satisfação, destacam-se o salário $(2,4)$ e a participação no processo de avaliação das atividades e dos programas do serviço (3).

Realizou-se a comparação dos escores de satisfação específicos para os diferentes profissionais da equipe de enfermagem. O menor escore médio de satisfação em relação às enfermeiras foi $(2,8)$, dos técnicos de en- fermagem, $(3,1)$ e dos auxiliares de enfermagem, $(3,3)$, referentes à Participação no serviço. Na comparação entre os membros da equipe de enfermagem, foi realizado o teste de Kruskal-Wallis e não foram observadas diferenças estatísticas significativas entre os escores de satisfação $(p=0,81)$.

Em relação às questões qualitativas de satisfação, observou-se que aproximadamente $80 \%$ dos sujeitos entrevistados gostam de estar com os pacientes, cuidar e ver sua melhora, seguida pelo bom relacionamento com os colegas de trabalho.

O que mais gera insatisfação na equipe (aproximadamente 64\%) são os aspectos relacionados às normas e regras institucionais e a desvalorização dos profissionais pela administração do hospital.

A grande maioria dos profissionais (95\%) acredita que o serviço pode ser melhorado com a realização de mais treinamentos e educação continuada e que a contratação de mais funcionários para os setores também iria proporcionar melhorias.

\section{Sobrecarga no trabalho}

Através dos dados obtidos da Escala de Avaliação IMPACTO-BR, verificou-se que o escore médio de sobrecarga dos profissionais foi 1,9, variando de 1,0 a 4,3. 0 grau médio de sobrecarga encontrava-se entre De forma alguma e Não muito, indicando índices relativamente baixos de sobrecarga. Os valores de sobrecarga obtidos, discriminando o impacto global e os fatores da escala encontram-se na Tabela 2.

TABELA 2: Média e desvio-padrão dos escores de sobrecarga geral e por fatores, de acordo com as respostas dos profissionais de um hospital psiquiátrico. Franca, São Paulo, 2013. ( $N=116)$

\begin{tabular}{lccc}
\hline \multicolumn{1}{c}{ IMPACTO-BR } & $\begin{array}{c}\text { Escore } \\
\text { médio }\end{array}$ & $\begin{array}{c}\text { Desvio } \\
\text { padrão }\end{array}$ & Variação \\
\hline Global & 1,9 & 0,7 & $1-4,3$ \\
Saúde física e mental (F1) & 1,7 & 0,7 & $1-4,0$ \\
Relacionamento da equipe(F2) & 2,1 & 0,8 & $1-4,8$ \\
Emocionais (F3) & 2 & 0,7 & $1-4,0$ \\
\hline
\end{tabular}

O fator de maior impacto foi efeito do trabalho no relacionamento da equipe, seguido pelo fator repercussões emocionais do trabalho. O fator de menor sobrecarga foi em relação aos efeitos sobre a saúde física e mental dos sujeitos.

Entretanto, as três variáveis de maior impacto foram o cansaço físico após o trabalho $(2,9)$, o receio da possibilidade de ser fisicamente agredido por algum paciente $(2,7)$ e o pensamento em mudar de campo de trabalho $(2,2)$.

A necessidade de procurar ajuda de algum profissional de saúde mental, devido a problemas emocionais decorrentes do trabalho $(1,2)$, foi a variável com escore 
de menor impacto de sobrecarga, entre as três menores variáveis, seguida pelo sentimento depressivo por trabaIhar com portadores de transtornos mentais $(1,3)$. O fato de ter contato com pessoas portadoras de transtorno mental, e isso afetar a vida social dos trabalhadores, foi a terceira menor variável de sobrecarga $(1,3)$.

Na análise do coeficiente de Correlação de Spearman, observou-se correlação negativa significativa a $5 \%$ dos escores de satisfação e sobrecarga ( $r=-0,4987)$.

Realizou-se, também, a Correção de Bonferroni para as comparações múltiplas, o que permite afirmar que todo o conjunto de resultados é significante a $5 \%$.

Assim, os escores de satisfação foram associados aos escores de sobrecarga, em cada domínio, tendo alguns resultados significativos, conforme discriminados na Tabela 3.

Realizou-se a comparação dos escores de impacto entre as três categorias da equipe de enfermagem. 0 escore médio de maior impacto nas enfermeiras foi 2,1 - o fator Repercussões emocionais do trabalho; nos técnicos de enfermagem, 2,2-Relacionamento da equipe; nos auxiliares de enfermagem, 2,0 - Relacionamento da equipe e as Repercussões emocionais do trabalho. No Teste de Kruskal-Wallis, não foram observadas diferenças estatísticas significativas entre os escores de impacto da equipe de enfermagem $(p=0,92)$.

Em relação às questões qualitativas de sobrecarga, os sujeitos foram questionados sobre os aspectos do trabalho que resultam em mais sobrecarga. Aproximadamente $80 \%$ afirmam que enfrentam problemas por ter poucos funcionários nos setores, tanto pelo número insuficiente de funcionários contratados pela instituição quanto pelas faltas e afastamentos dos colegas de trabalho.

O aumento do número de funcionários foi considerado condição de menor sobrecarga no serviço por aproximadamente (75\%) dos sujeitos e, em seguida, a realização do trabalho em equipe.

Quanto aos aspectos do trabalho que gostariam de mudar para diminuir a sobrecarga, disseram que seria a contratação de mais funcionários e o aumento do salário (67\%).

\section{DISCUSSÃo}

\section{Satisfação no trabalho}

Os dados obtidos revelam que os profissionais estudados apresentam níveis intermediários de satisfação, situados na indiferença. Estes resultados apontam para a necessidade de revisão dos projetos organizacionais, na busca de melhorar o serviço.

Compreende-se que os profissionais estudados estão mais satisfeitos com os relacionamentos interpessoais no ambiente de trabalho e insatisfeitos quanto a sua participação nas decisões da sua unidade de trabalho.

Em outros serviços de saúde, também verificou-se que as relações interpessoais aparecem como fator de relevância no ambiente de trabalho, a atuação em equipe é sentida como ponto de apoio e de alívio ${ }^{14}$.

Entre os escores de menor satisfação, os participantes sentem-se insatisfeitos com os benefícios e o salário oferecido pela instituição, semelhante a outros estudos $^{15,16}$ e com a sua participação nas atividades e programas do serviço, sugerindo que os aspectos de natureza organizativa estão entre os mais insatisfatórios do serviço.

A questão salarial é citada na literatura nacional e internacional como uma das causas de insatisfação e de preocupação entre os profissionais ${ }^{17}$.

Trabalhar em equipe é uma ótima maneira para obter bons resultados, dentro das organizações. Os gestores e administradores precisam confiar e respeitar as ações dos membros da equipe. Para as empresas obterem bons resultados do trabalho oferecido e para manterem-se no mercado, precisam estimular o trabaIho de forma integrada, com responsabilidade coletiva para obter bons resultados ${ }^{18}$.

Segundo os sujeitos do presente estudo, há poucas expectativas de promoção no serviço. A capacitação e o aperfeiçoamento em saúde mental não são considerados, desmotivando, dessa forma, a busca por melhor formação e estudos na área de saúde mental.

A partir dos resultados apresentados, observa-se a necessidade da instituição rever alguns pontos da sua administração com vistas a proporcionar melhores

TABELA 3: Coeficientes de Correlação de Spearman ${ }^{(*)}$ para os escores de satisfação e de impacto no trabalho, em serviço psiquiátrico. Franca, São Paulo, 2013 (N=116)

\begin{tabular}{lccccc}
\hline \multicolumn{1}{c}{ Impacto } & $\begin{array}{c}\text { Satisfação } \\
\text { qualidade } \\
\text { do serviço }\end{array}$ & $\begin{array}{c}\text { Participação } \\
\text { no serviço }\end{array}$ & $\begin{array}{c}\text { Satisfação } \\
\text { condições de } \\
\text { trabalho }\end{array}$ & Relacionamentos & Geral \\
\hline $\begin{array}{l}\text { Sobre a saúde física e } \\
\text { mental }\end{array}$ & $-0,3973$ & $-0,3553$ & $-0,4681$ & $-0,3879$ & $-0,4609$ \\
$\begin{array}{l}\text { Relacionamento da } \\
\text { equipe }\end{array}$ & $-0,4716$ & $-0,4126$ & $-0,5688$ & $-0,3233$ & $-0,5274$ \\
$\begin{array}{l}\text { Emocional } \\
\text { Global }\end{array}$ & $-0,3691$ & $-0,293$ & $-0,3654$ & $-0,2046$ & $-0,3626$ \\
\hline
\end{tabular}

${ }^{(*)}$ Correlações significantes ao nível de 5\%, com correção para comparações múltiplas. 
condições de trabalho para a equipe e permitir a participação mais efetiva desses profissionais no serviço, o que motivará os funcionários.

Percebeu-se, também, que a satisfação em relação ao fator Condições de trabalho é menor na equipe de enfermagem, se comparada aos demais profissionais da equipe multidisciplinar.

Entre os aspectos que podem ter influenciado a menor satisfação das condições de trabalho, entre os membros da equipe de enfermagem, destaca-se o baixo salário da classe, muitas vezes insuficiente para a manutenção da vida pessoal e de seus familiares, levando-os a procurar outras formas de sustento, com duplas ou até em alguns casos, triplas jornada de trabalho.

Foram identificados alguns fatores que levam à insatisfação no trabalho ${ }^{19}$. A satisfação é sentida pela autonomia, bom salário e boas condições de trabalho, capacitação e treinamentos, realização profissional e expectativa de ser promovido; por outro lado, a insatisfação por falta de apoio dos superiores, falta de profissionalismo e coleguismo, setor de trabalho inadequado, falta de materiais e equipamentos, baixo salário, número insuficiente de funcionários sobrecarregando a equipe, estresse no ambiente de trabalho, falta de interação entre a equipe, falta de reconhecimento, não ser valorizado, insegurança quanto à estabilidade no emprego entre outros.

Carga horária excessiva, baixa remuneração e ausência de horas de lazer afetam negativamente a saúde física e mental dos trabalhadores, sendo, dessa maneira, necessário repensar nos motivos que levaram os profissionais de enfermagem a sentirem-se mais insatisfeitos com as condições de trabalho quando comparados aos demais membros da equipe multiprofissional ${ }^{15}$.

A leitura das respostas às questões qualitativas da Escala SATIS-BR revelou, dentre os fatores mais frequentemente apontados como fonte de satisfação para os profissionais da instituição estudada, o contato e o cuidado oferecido aos pacientes, seguido pelo relacionamento harmonioso com os colegas de trabalho. Os aspectos organizacionais, como as normas e regras, impostas verticalmente, foram apontados como os fatores que mais contribuíram para menor satisfação, seguido pela insatisfação quanto à valorização profissional.

Os participantes do estudo acreditam na possibilidade de melhora do serviço através da realização de treinamentos, bem como a contratação de mais funcionários, que iria resultar em menor sobrecarga.

\section{Sobrecarga no trabalho}

Considerando-se que o hospital psiquiátrico possui características favoráveis à sobrecarga dos profissionais, pelo fato de manter contato direto com pacientes em surtos psicóticos e estados clínicos graves, esperava-se encontrar escores de sobrecarga elevados. Entretanto, não foi o encontrado nesta instituição.
Estudando-se trabalhadores de enfermagem de um hospital universitário do Rio Grande do Sul, observou-se que distúrbios psíquicos menores ocorrem em $18,7 \%$ dos profissionais e que esses são mais frequentes entre os trabalhadores que sofrem alta exigência de trabalho ${ }^{20}$.

No presente estudo, diante dos resultados relativamente baixos, referentes à sobrecarga, cogita-se que os profissionais encontraram formas ou estratégias de enfrentamento para diminuí-la. Tais estratégias foram identificadas em outro estudo que, investigando formas de enfrentamento contra o estresse entre a equipe de enfermagem de um pronto-atendimento, encontrou o evitamento, onde os profissionais procuravam esquecer dos fatores estressores, adiavam confrontos ou bloqueavam suas emoções; eles falavam sobre o problema e negociavam alternativas; participavam de atividades religiosas, esportivas e de lazer, sendo essas utilizadas para aliviar o grau de tensão no trabalho ${ }^{21}$.

Na presente pesquisa, observou-se significativa satisfação dos profissionais em relação ao fator Relacionamentos no trabalho, supondo-se que a ajuda dos colegas de trabalho contribui para amenizar a sobrecarga ocupacional, influenciando o resultado de baixo escore de sobrecarga encontrado.

Percebe-se então, a partir desses resultados, que os profissionais da equipe em estudo sofrem mais sobrecarga através dos efeitos do trabalho na qualidade da saúde do trabalhador e menos sobrecarga através dos efeitos do trabalho sobre a saúde física e mental dos profissionais.

Pesquisando-se profissionais de serviços de saúde mental, evidenciou-se que estratégias de suporte social, ou seja, a busca de apoio e ajuda através do relacionamento com outras pessoas, como familiares, amigos ou colegas de trabalho, ajudam a diminuir a sobrecarga decorrente do contato cotidiano com usuários em sofrimento mental22.

É necessário que os gestores de saúde contribuam para minimizar a sobrecarga dos profissionais, proporcionando, como consequência, um melhor atendimento e maior segurança do paciente $e^{23}$.

Sabe-se que quando existem meios que garantam o bem-estar dos profissionais no seu local de trabalho, eles se tornam mais motivados e produzem com maior qualidade ${ }^{24}$.

Acredita-se que quanto melhor for a percepção das instituições e da equipe de enfermagem em relação à necessidade da manutenção e da prevenção da saúde mental, melhores serão as condições de trabalho e de qualidade de vida desse trabalhador, uma vez que esses poderão ficar mais satisfeitos, felizes e realizados, pessoal e profissionalmente.

As organizações devem estimular, incentivar e motivar seus profissionais, criar um ambiente satisfatório e saudável aos trabalhadores ${ }^{25}$. 
Em relação aos escores de menor impacto, estão a necessidade de procurar ajuda de um profissional de saúde mental, sentir-se deprimido por trabalhar com portador de transtorno mental e, por último, o contato com os usuários afetar a vida social dos participantes, demonstrando, dessa forma, que o estado emocional desses profissionais sofre menos impacto na instituição em estudo.

Com a análise dos escores da Escala SATIS-BR e IMPACTO-BR, verificou-se que todo o conjunto de resultados é significante, sugerindo que quanto maior o grau de sobrecarga, menor é o grau de satisfação.

O fato de lidar com o sofrimento orgânico, emocional ou social requer dos profissionais envolvidos o desenvolvimento de competências interpessoais ${ }^{26}$, sugerindo como ideal o acompanhamento psicológico dos profissionais, no intuito de desenvolverem estratégias de adaptação e manejo para atuar com os usuários.

No presente estudo, verificou-se que os profissionais de enfermagem sofrem maior impacto do que os demais membros da equipe e o Relacionamento da equipe é o fator que mais os sobrecarregam.

Medidas profiláticas de promoção e prevenção da saúde do trabalhador precisam ser iniciadas ainda nos cursos profissionalizantes e de formação superior, para que a partir desse momento os futuros profissionais possam ter consciência da importância da manutenção de sua saúde.

$\mathrm{Na}$ leitura das respostas referentes às questões qualitativas da Escala IMPACTO-BR, notou-se que a equipe multidisciplinar sente-se sobrecarregada e insatisfeita com o número de funcionários da instituição, sugerindo a contratação de funcionários, o trabalho em equipe e o aumento de salário - aspectos que proporcionariam menor sobrecarga.

Ficam, ainda, questões que merecem atenção futura: onde está a preocupação desses funcionários com a qualidade da assistência prestada? Será um viés do instrumento aplicado?

Tendo em vista a sobrecarga vivenciada pela equipe desse estudo em relação aos aspectos organizacionais, torna-se relevante a reflexão e a busca de alternativas com o intuito de proporcionar melhorias no ambiente institucional, tais como condições de trabalho, salário, benefícios, promoções e incentivos ao crescimento profissional e aperfeiçoamento contínuo na área de saúde mental, realização de mais treinamentos, implementação de programas de prevenção e promoção da saúde dos trabalhadores.

\section{CONCLUSÕES}

Os resultados mostraram que os profissionais da equipe multiprofissional apresentam baixos níveis de satisfação, sentem-se mais satisfeitos quando em contato com os usuários e no relacionamento com os membros da equipe.
Baixos índices de sobrecarga foram identificados, embora a contratação de funcionários, o aumento do salário, a valorização profissional e o trabalho em equipe aparecessem como aspectos necessários para a melhoria do serviço.

A equipe de enfermagem apresenta menor satisfação quanto às condições de trabalho e sofre maior impacto no trabalho quando comparada aos demais profissionais da equipe multiprofissional.

No entanto, os resultados alertam para as condições de trabalho, a participação no serviço e os aspectos de natureza organizativa, estando entre os que mais geram insatisfação no serviço.

Em vista desses resultados, percebe-se a necessidade da revisão dos projetos institucionais, na busca de melhores benefícios, incentivos e valorização profissional. Programas de promoção e prevenção da saúde do trabalhador precisam ser implantados na presente instituição. O investimento em capacitação na área de saúde mental da equipe e a contratação de mais funcionários também podem ser considerados.

\section{REFERÊNCIAS}

1.Pereira JA, Alves KS, Silva DCC. A qualidade de vida no trabalho com vistas à promoção de saúde física e emocional do trabalhador no setor calçadista de Franca-SP. Franca (SP): Editora Uni-FACEF; 2008. 2.Martinez MC, Paraguay AIBB. Satisfação e saúde no trabalho: aspectos conceituais e metodológicos. Cad psicol soc trab. 2003; 6(1): 59-78.

3.Elias MA, Navarro VL. A relação entre o trabalho, a saúde e as condições de vida: negatividade e positividade no trabalho das profissionais de enfermagem de um hospital escola. Rev Latino-Am Enfermagem. 2006; 14(4):517-25.

4.Minayo MCS, Hartz ZMA, Buss PM. Qualidade de vida e saúde: um debate necessário. Ciênc saúde coletiva. 2000; 5(1):7-18.

5.Heloani JR, Capitão CG. Saúde mental e psicologia do trabalho. São Paulo Perspec. 2003; 17(2):102-8.

6.Silva RM, Beck CLC, Guido LA, Lopes LFD, Santos JLG. Análise quantitativa da satisfação profissional dos enfermeiros que atuam no período noturno. Texto contexto - enferm. 2009; 18(2):298305.

7.Rosa C, Carlotto MS. Síndrome de Burnout e satisfação no trabalho em profissionais de uma instituição hospitalar. Sociedade Brasileira de Psicologia Hospitalar. 2005; 8(2):1-15.

8.Matsuda LM, Évora YDM. Ações desenvolvidas para a satisfação no trabalho da equipe de enfermagem de uma UTI-adulto. Ciência, Cuidado e Saúde Maringá. 2006; 5:49-56.

9.Melo MV, Silva TP, Novais ZG, Mendes MLM. Estresse dos profissionais de saúde nas unidades hospitalares de atendimento em urgência e emergência. Cadernos de Graduação - Ciências Biológicas e da Saúde Facipe. 2013; 1 (2): 35-42.

10.Farias SMC, Teixeira OLC, Moreira W; Oliveira MAF, Pereira MO. Caracterização dos sintomas físicos de estresse na equipe de pronto atendimento. Rev esc enferm USP. 2011; 45(3):722-9. 11.Secco IAO, Robazzi MLCC, Souza FEA, Shimizu DS. Cargas psíquicas de trabalho e desgaste dos trabalhadores de enfermagem de hospital de ensino do Paraná, Brasil. Revista Eletrônica Saúde Mental Álcool e Drogas. 2010; 6(1):1-17.

12.Bandeira M, Pitta AM F, Mercier C. Escalas da OMS de avaliação da satisfação e da sobrecarga em serviços de saúde mental. Jornal Brasileiro de Psiquiatria. 2000; 48(6):233-44. 
13.Siegel S, Castellan Júnior NJ. Estatística não paramétrica para as ciências do comportamento. $2^{\text {a }}$ ed. Porto Alegre (RS): Artmed; 2006 14.Silva EA, Costa II. Saúde Mental dos trabalhadores em saúde mental: estudo exploratório com os profissionais dos Centros de Atenção Psicossocial de Goiânia/GO. Psicologia em Revista. 2008; 14(1):83-106.

15.Santos AM, Cardoso DAJ, Vieira DPB, Araújo FC, Farias HS, Mota SP et al. Análise dos níveis de satisfação de trabalhadores de saúde mental de um hospital público de referência psiquiátrica em Belém (PA). Revista Baiana de Saúde Pública. 2011; 35(4):813-25. 16.Santos MCL, Braga VAB, Fernandes AFC. Niveis de satisfação dos enfermeiros com o seu trabalho. Rev enferm UERJ. 2008; 16(1):101-5. 17.Versal GLGS, Matsuda LM. Satisfação profissional da equipe de enfermagem intensivista de um hospital de ensino. Rev enferm UERJ. 2014; 22(3):409-15.

18.Torres A. Trabalho em equipe [monografia]. Brasília (DF): Universidade de Brasília; 2011.

19.Cardoso RAS, Almeida ML. Motivação e satisfação da equipe de enfermagem: Percepção de profissionais de uma unidade clínica de um hospital público de Foz do Iguaçu. In: IV SEECEL- Ensino, Pesquisa e Extensão em foco: com a palavra os profissionais das Letras, da Pedagogia e da Enfermagem; 2009; Foz do Iguaçu, Brasil. Foz do Iguaçu (PR); 2009. p.1-15.
20. Kirchhof ALC, Magnago TSBS, Camponogara S, Griep RH, Tavares JP, Prestes FC et al. Condições de trabalho, características socio-demográficas e distúrbios psíquicos menores em trabalhadores de enfermagem. Texto contexto - enferm. 2009; 18(2):215-23.

21.Calderero ARL, Miasso Al, Webster CM. Estresse e estratégias de enfrentamento em uma equipe de enfermagem de Pronto Atendimento. Revista Eletrônica de Enfermagem. 2008; 10(1):51-62. 22.Santos AFO, Cardoso CL. Profissionais de saúde mental: estresse, enfrentamento e qualidade de vida. Psicologia Teoria e Pesquisa. 2010; 26(3):543-8.

23 Novaretti MCZ, Santos EV, Quitério LM, Daud-Gallotti R. Sobrecarga de trabalho da Enfermagem e incidentes e eventos adversos em pacientes internados em UTI. Rev. Bras. Enferm. 2014; 67(5): 692-9.

24. Clein C, Tonello R, Pessa SLR. Influência do ambiente de trabaIho na saúde física e emocional do trabalhador:estudo ergonômico em uma fábrica de máquinas industriais. Revista ADMpg Gestão Estratégica.2014; 7(1): 53-9.

25.Marçal CZ, Melo FP, Nardi A. Satisfação no trabalho: um estudo de caso numa empresa terceirizada. Rev Científica Eletrônica UNISEB. 2013; 1(1):20-35.

26.Santos AFO, Cardoso CL. Profissionais de saúde mental: manifestação de stress e Burnout. Estudos e Psicologia. 2010; 27(1):67-74. 